\title{
A novel area coverage management scheme for sensor network with mobile sensor nodes
}

\author{
Archana Mishra $^{1 *}$, Sudhansu Sekhar Singh ${ }^{1}$, Saikath Bhattacharya ${ }^{1}$ and Prasant Kumar Pattnaik ${ }^{2}$ \\ ${ }^{1}$ School of Electronics Engineering; ${ }^{2}$ School of Computer Engineering, KIIT University, Bhubaneswar, 751024, India \\ archana.mishra018@gmail.com,ssinghfet@kiit.ac.in,saikath.bhattacharya@gmail.com
}

\begin{abstract}
Wireless sensor networks (WSNs) are with sensor nodes that can sense certain physical characteristics and features to capture environmental information such as temperature, pressure, humidity sound, motion etc. WSNs are capable of forming a self-organizing network. A self organizing network is the one that gets up automatically without any form of manual setup or configuration. Again the deployment of these networks to cover the desired area in specific environment is a challenge. However, many works have been cited about this issue of area coverage problem with static sensor nodes but automatic configuration of sensor node with flexible mobility and less energy consumption are the matter of prime consideration. Again, suitable deployment schemes are crucial for achieving best link performance. This paper proposes a model based on artificial potential field along with rank of sensor node that decides which node will participate in mobility to cover the specified area. Our analytical study is based on Voronoi diagram and artificial potential fields which bring out significant outcomes.
\end{abstract}

Keywords: Self Deployment, Area coverage, Rank, Voronoi polygon, Energy optimization.

\section{Introduction}

The sensor nodes can sense, assess, and assemble information from the environment and, based on some local decision process, they can transmit the sensed data to the user. Sensor nodes are low power devices set with one or more sensors, a processor, memory, a power supply, a radio, and an actuator of the environment. Battery is the main power source in a sensor node. Depending on the application and the type of sensors used, actuators may be built-in in the sensors. Deployment of wireless sensor node (Howard \& Mataric, 2002) is the difficult task in case of the hostile environment, say dense forest, where the direct access by the human being may be tedious and time consuming. In this scenario, the deployment of node may be undertaken through the helicopters by scattering the nodes randomly. However, if some self movement may be taken place to the sensor nodes, they might move freely in order to spread over the area and sense the environment more accurately and timely.

Wireless sensor networks have found immense use in all the important communication application domains like industrial, military, medical, home, office \& pervasive computing in the past two decades. The usage of sensor networks is rapidly growing with small sensor size and easy deployment techniques. We can easily expand and shrink such network. This makes the wireless sensor network more flexible. In a wireless sensor network, a number of nodes are deployed in a region and connected to each other by means of wireless communication. This network has certain limitations such as: Limited energy supply, Limited storage, Wireless communication, Limited processing. Though WSN has some limitations, it has many applications such as: Environmental Monitoring to detect environmental changes in plains, forests, industrial sensing and diagnostics, Infrastructure protection etc. Flexibility in network coverage and data acquisition improves enormously by providing mobility to sensor

(C) Indian Society for Education and Environment (iSee)

"Wireless sensor networks"

http://www.indjst.org

nodes. Some of the important applications in this segment are battlefield awareness to detect and gain as much information as possible about enemy movements, explosions and other phenomena of interest, Biological and chemical attack detection. The complexity of the network increases further with added mobility condition for the nodes. With various mobility conditions the performance of the network is analyzed and optimized performance for energy and coverage is the prime objective for any mobile wireless sensor network.

Self-Deployment of wireless sensor networks (Sameera Poduri \& Gaurav, 2004) is essential in some specific applications such as the unknown, hostile environment like dense forest, a burning house, spying terrorist and enemy locations, actually the places where much risk is involved in gathering information in incarnate manner. Random deployment of sensor nodes may give rise to unnecessary loss of excess energy, and thus network lifetime becomes shorter. It is required to find the optimal placement of the nodes so that the network can be able to collect maximum information while minimizing the energy usage required for the network (Shafaq Chaudhary et al., 2006). The aggregation of sensor nodes should be such that strong data communication paths must exist to allow for seamless gathering and reporting of sensed information. Moreover, some redundancy in these data paths is very much needed, since it imparts fault tolerance to the network.

\section{WSN Vs MWSN}

The major elements of WSN are the sensor nodes and the base stations. In fact, they can be distracted as the "sensing cells" and the "brain" of the network, respectively. Generally, sensor nodes are deployed in a selected area by an authority and then, automatically form a network through wireless communications. A WSN is a large network of resource-constrained sensor nodes with multiple predetermined functions, such as sensing and processing, to fulfill different application objectives. 
Sensor nodes of homogeneous or heterogeneous type can be deployed randomly or at pre-determined locations using a deterministic scheme (Cardei \& Wu, 2006). Sensor nodes are static most of the time, whereas mobile nodes can be deployed according to application requirements known as Mobile wireless sensor network (MWSN). In these type applications mobility may be redundant and overweight. Sensor nodes keep monitoring the network area after being deployed. After an event of interest occurs, one of the surrounding sensor nodes can detect it, generate a report, and transmit the report to a BS through multi hop wireless links. Association can be carried out if multiple surrounding nodes detect the same event. In this case, one of them generates a final report after associating with the other nodes. The Base Station can process the report and then forward it through either high-quality wireless or wired links to the external world for further processing (Wang et al., 2004).

\section{Coverage Issue}

The coverage Problem (Cardei \& Du, 2005) is such an active issue of WSN that determines how efficiently the sensor network is being covered by a set of sensor nodes. It ensures that a specific region is covered by one or more sensor nodes. It may be broadly classified into three main categories: a) Area Coverage Problem (Carle \& Simplot, 2004) where the sensor nodes are deployed to cover a specific area or region. b) Target Coverage Problem (Thai \& Li, 2005) where the sensor nodes are deployed to cover a specific set of targets or points. c) Coverage dealing with the determination of the maximum support /breach path (Meguerdichian et al., 2001)

\section{Challenges}

Wireless mobile Sensor Nodes while covering the specific area may suffer from followings: a) Wireless mobile sensor nodes free to move in order to cover area so the topology of a sensor network changes dynamically and frequently. b) Sensor nodes are limited in power, computational capacities, and memory. c) WMSN deals with real world problems where the sensed data must be delivered within fixed time limits.

However, this high redundancy of sensor nodes can lead to excessive energy wastage and also the maintenance of batteries of the sensor nodes is not a simple task, thereby making energy conservation a critical issue of WSN. So, while covering the specific targets, several issues like minimum participation of sensor nodes, minimum consumption of energy, etc must be taken into consideration in order to achieve much efficient coverage.

\section{Proposed model}

We have considered an Area coverage problem with the given Area to be cover (or Area of Interest) denoted by ' $A$ ' on a two-dimensional plane which is to be monitored using ' $N$ ' number of Mobile Sensor Nodes. Each Node is assumed to have equal Sensing range $\left(S_{R}\right)$ and equal Communication Range $\left(C_{R}\right)$. The sensing quality of a node is uniform throughout its sensing range. Each node must be having communication, computation and also locomotion capabilities. All these nodes are able to move at any direction within the area of interest. These nodes should deploy themselves as the result coverage will be maximized, keeping the communication between them to a certain level with minimum energy consumption. The area coverage will be increased when the movement of the node will be done. But energy consumption for movement of a node is many times

Fig. 1(a). All the nodes are left together at one point in the obstacle and the area of interest

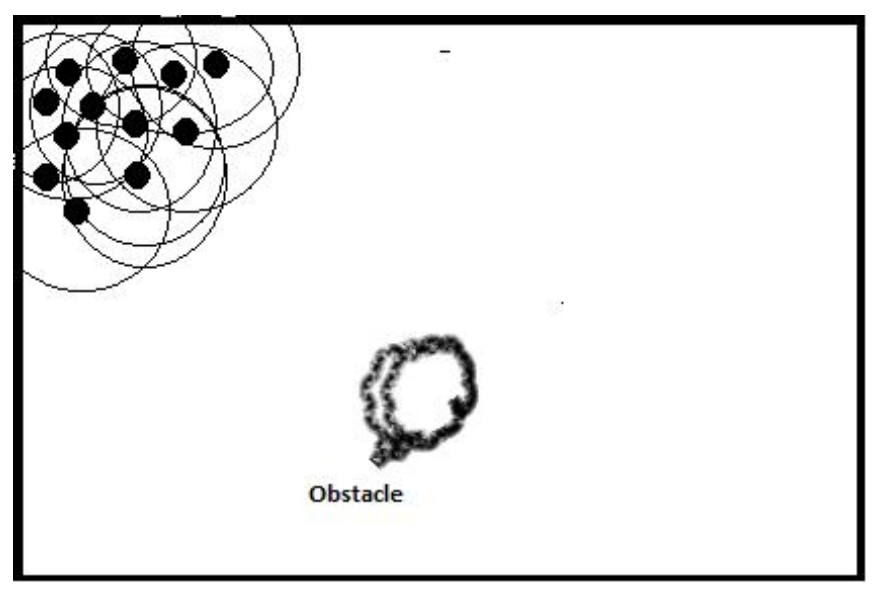

Fig. 1(b). The repulsive force upon the nodes due to neighboring nodes

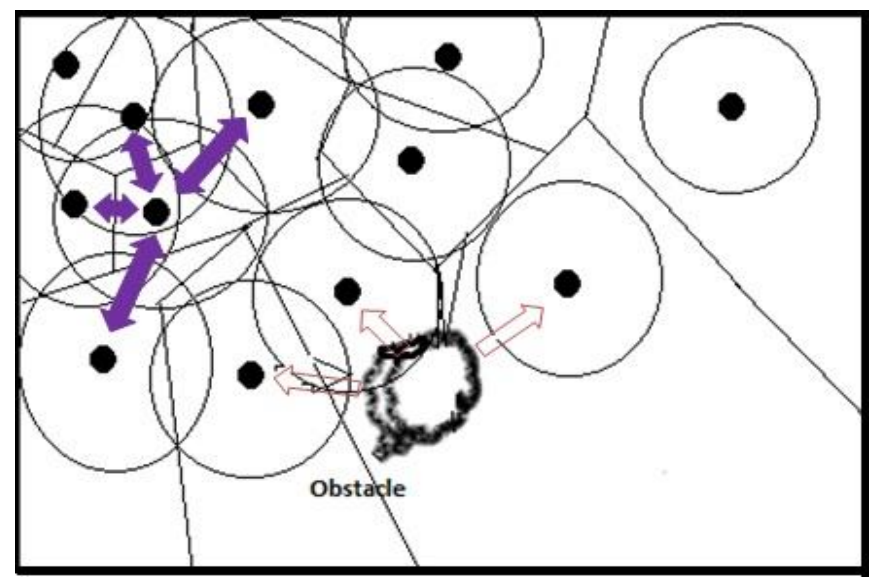

higher than the communication between two nodes. Therefore movement is to be done for most required conditions. The nodes which are within the communication range of a node are called it's neighboring nodes. The number of neighboring nodes for proper communication is decided before the deployment process starts. There might be one or more obstacles in the environment.

Considering all the above assumptions the problem is formulated as follows: In a given area ' $A$ ' with obstacles using ' $N$ ' number of nodes how to maximize the coverage area to gather optimum information with minimum energy consumption. Here the nodes will take their own 
Fig. 2. Voronoi diagram of a node showing the attractive force and repulsive force

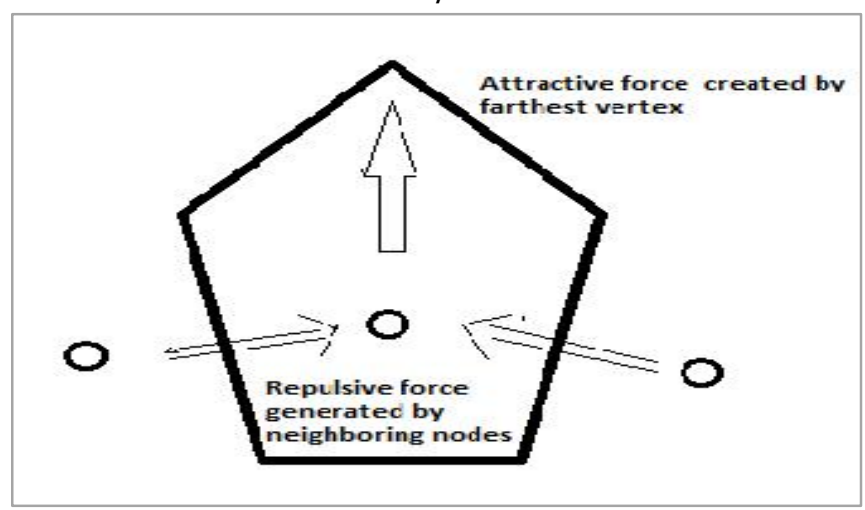

Fig. 3(a). Deployment of nodes in an area of interest randomly

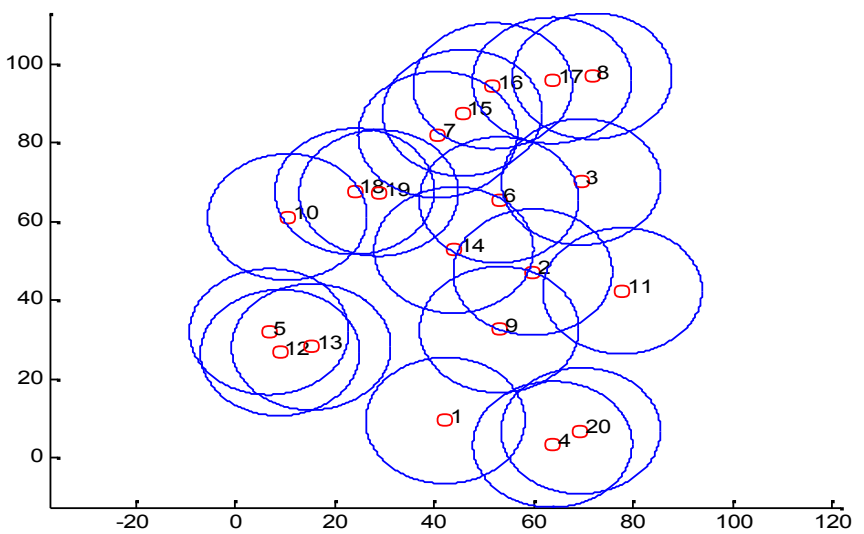

Fig. 3(b). Deployment of nodes in the area of interest using

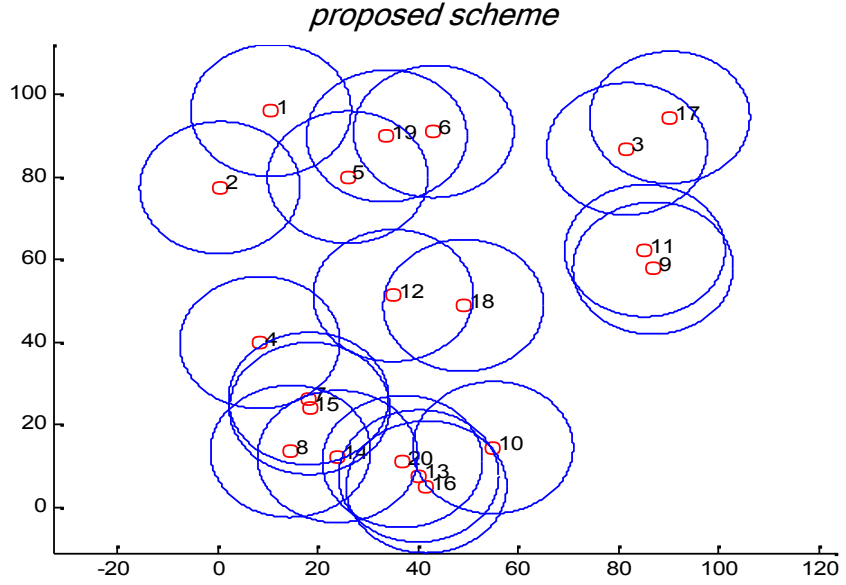

movement decision by the information available with them and following terminologies are used by our algorithm.

Area of the voronoi polygons

The area of each voronoi polygon is estimated. The density of the nodes is higher where the area of the polygons is less and the reverse is true. Taking this into account the first step of movement is decided.

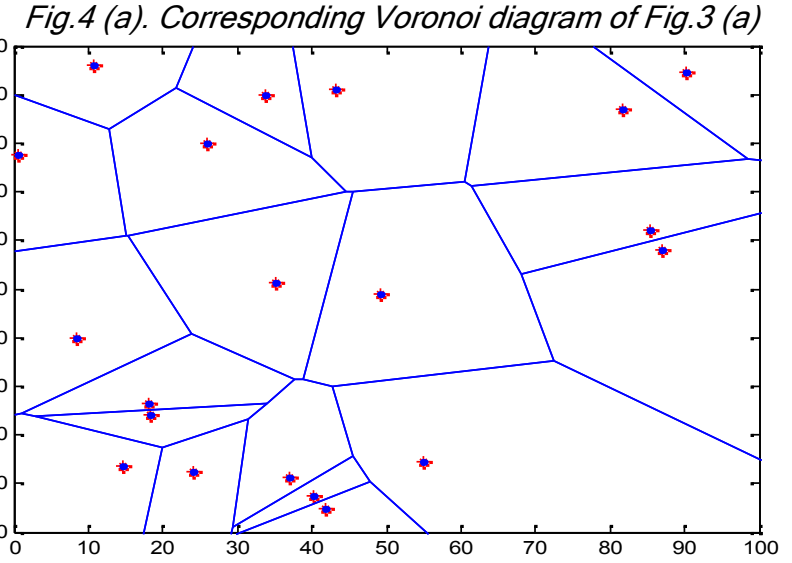

Fig. 4(b). Corresponding Voronoi diagram of Fig.3 (b)

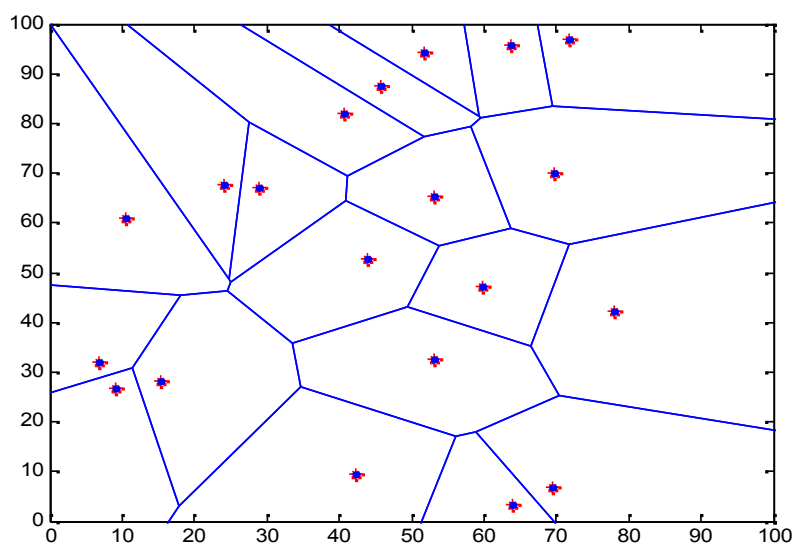

Fig.5 (a).Energy diagram with distance in

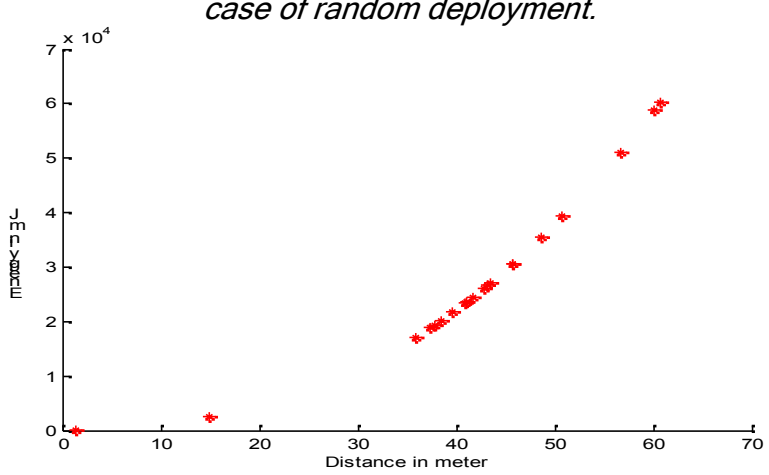

Fig.5 (b).Energy diagram of proposed scheme.

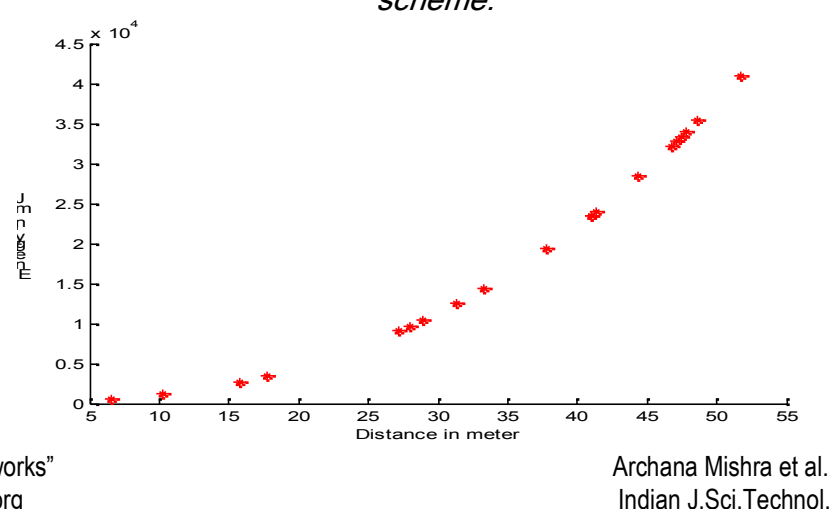




\section{Sensing area}

Sensing area is the area around the node within which it can sense the environment.

Rank

Initially the mobile nodes will be provided with certain amount of energy. An energy threshold is specified for the nodes after reaching that threshold level the node can't move. Rank of a node is the ratio of the energy content of that node at that point of time to the Threshold energy .Nodes having higher energy is having higher rank. After each steps of movement the rank of the nodes changes. Then the next step of movement is decided.

$\mathrm{R}_{\mathrm{i}}=\mathrm{e}_{\mathrm{i}} / \mathrm{e}_{\mathrm{t}}$

Where,

$\mathrm{R}_{\mathrm{i}}=$ Rank of $\mathrm{i}^{\text {th }}$ node

$\mathrm{e}_{\mathrm{i}}=$ energy content of $\mathrm{i}^{\text {th }}$ node at that point of time

$\mathrm{e}_{\mathrm{t}}=$ specified energy threshold

Our scheme

Step-1: The sensor nodes are left within an unknown environment containing an obstacle as shown in Fig. 1(a). Then the nodes are subjected to the movement repulsing each other and the walls of the Area of Interest (AOI). The walls behave as the obstacles and create repulsive force .The repulsive force is taken from the potential field based technique (Howard et al., 2002).

Analytical model

Our Scheme includes following procedures namely fixing the direction of movements, energy check and stability check are discussed below:

Step-2: Fixing the direction of movement: Nodes are left at one place together in the AOI (Area of Interest). $\mathrm{N}=$ number of nodes. They are subjected to a random movement inside the $\mathrm{AOI}$. Each of the node creates voronoi polygon around itself taking the bisector of the distance between the node and it's neighboring nodes. Area of each polygon is calculated. The Density of the nodes is high where areas of the voronoi polygons are smaller. Density is low where area of polygons is larger.

If $\mathrm{V}<<<\mathrm{pi}\left(\mathrm{S}_{\mathrm{R}}\right)^{2}, \mathrm{D}$ is high and reverse is true.

Where

$\mathrm{V}=$ Area of voronoi polygon

$\mathrm{D}=$ density

Step-3: Expected density is calculated by

$\mathrm{U}\left(\mathrm{C}_{\mathrm{R}}\right)=\left(\mathrm{N} \cdot \mathrm{pi} \cdot \mathrm{C}_{\mathrm{R}}{ }^{2} / \mathrm{A}\right)$

Where $N=$ number of Nodes, $C_{R}=$ communication range, $A=$ area of $A O I$

The difference between the actual density (D) and the expected density $U\left(C_{R}\right)$ decides the movement .After first step of movement ,The second step of movement is done like this .If distance of a node to any of the vertices of the polygon $r \ll S_{R}$, movement is done iteratively till $r \rightarrow S_{R}$

Step-4: Energy check: The energy content of the nodes (Nojeong \& Pramod, 2005) is checked at the end of each and every step. Initially all the nodes are provided with equal amount of energy. After each step the nodes is left with different amount of energy. So initially all the nodes are having Rank ' 1 '.once they start movement, the distance covered by each node is different after a certain

Fig. 6(a). Node level energy diagram in case of random deployment

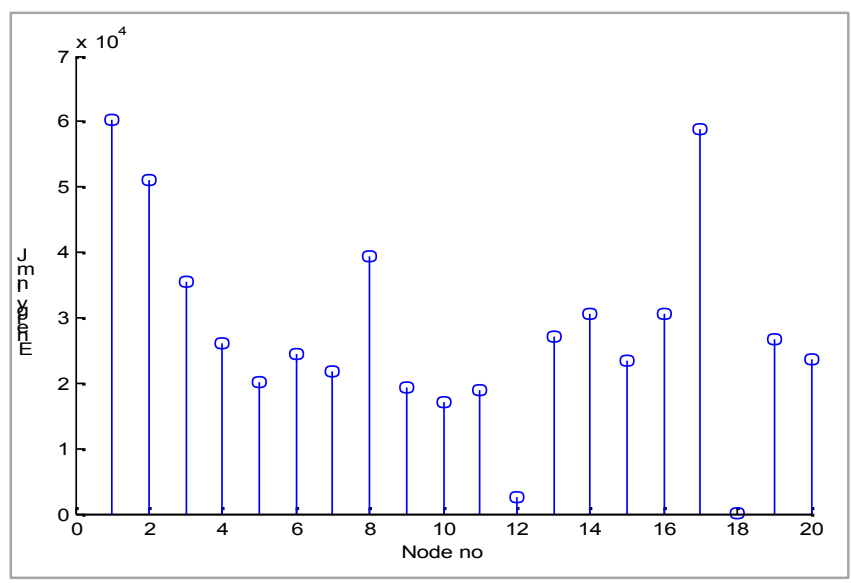

Fig. 6(b). Node level energy diagram in case of proposed scheme.

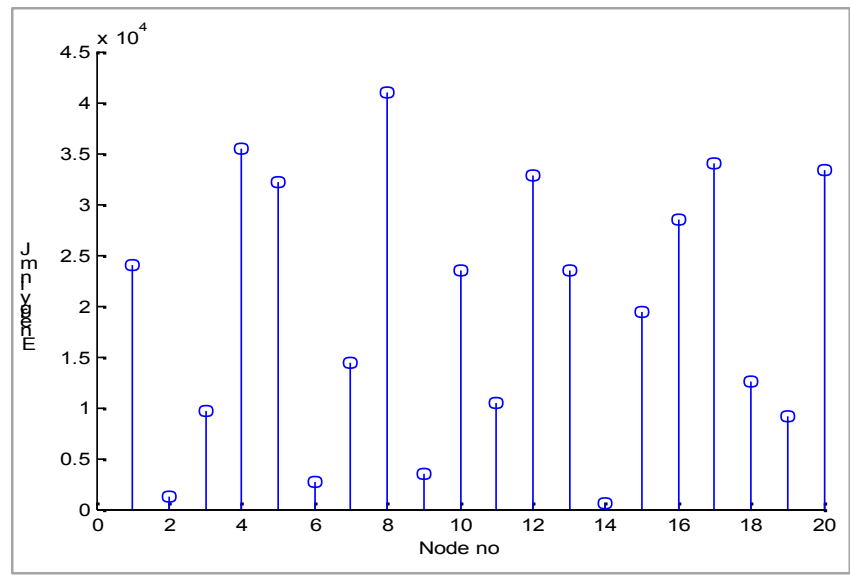

Fig. 7.Percentage coverage of the whole network with $N=20$

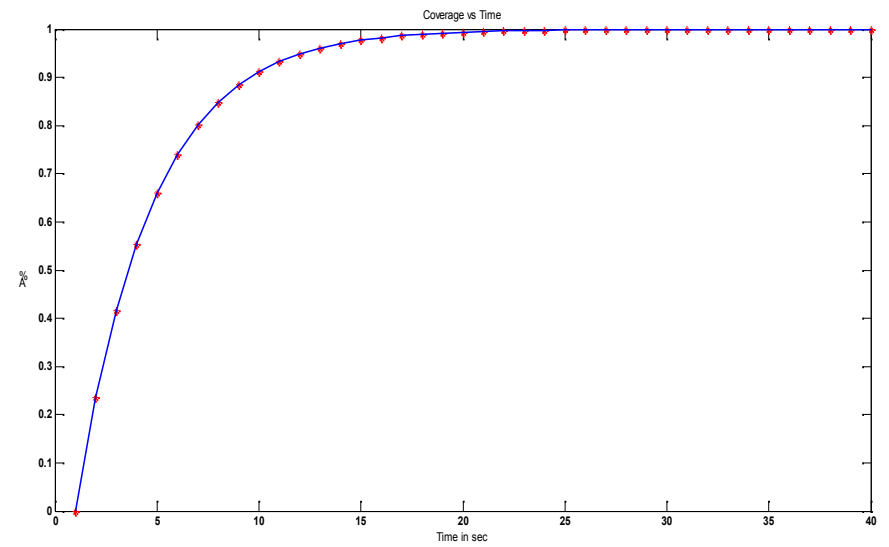

time interval. So the energy content of every node decreases differently.

As we are taking random mobility condition each node doesn't cover equal distance in equal time interval. So it
Research article

(C)Indian Society for Education and Environment (iSee)
"Wireless sensor networks" http://www.indjst.org
Archana Mishra et al. Indian J.Sci.Technol. 
doesn't depend upon time .we has considered here the step. After each step (round) the energy is calculated. Energy of a node ' $i$ ' at time step ' $n$ ' is calculated using the equation given below

$E(n, i)=e \operatorname{com}(n, i)+e \operatorname{comp}(n, i)+e \operatorname{move}(n, i)$

where Energy consumption due to communication

e $\operatorname{com}(n, i)=\left(d(i)^{\wedge} a\right)^{\star}\left(E\right.$ send $\left.+\left(K^{n *} E r e c v\right)\right)$

$\mathrm{d}(\mathrm{i})=$ distance travelled by node ' $\mathrm{i}$ '

a=attenuation constant $=2.5$

Esend $=$ Erecv $=1 \mathrm{~mJ} / \mathrm{m}$

$\mathrm{K}^{\mathrm{n}}$ =number of nodes, from which the node 'i'is receiving

the signals at step $n$.

Energy consumption due to computation:

e comp $(n, i)=\left(K^{n}+1\right)^{*} m^{*}$ Ecomp

where $\mathrm{m}=$ number of computations

Ecomp $=0.1 \mathrm{~mJ} / \mathrm{m}$

Energy consumption due to movement:

e move $(n, i)=d(i)^{*}$ Emove

where Emove $=50 \mathrm{~mJ} / \mathrm{m}$

After calculating the Rank, it is decided which node is the best to move to that location accordingly.

Step-5:Stability check: A threshold energy level is fixed initially. During energy check the node, whose energy content falls below the threshold limit that comes to equilibrium position. In this way the whole network stops movement and equilibrium is achieved.

Algorithm:

//All nodes are having initial position $\left(\mathrm{x}_{0}, \mathrm{y}_{0}\right)$

Number of nodes $\mathrm{N}$

Sensing Range $S_{R}$

Communication Range $\mathrm{C}_{\mathrm{R} / \mathrm{I}}$

Step-1:

//All nodes are left to Random movements Position of $i^{\text {th }}$ node is shifted to $\left(x_{i}, y_{i}\right), i=1,2 \ldots . . N / /$

Step-2:

Calculate Area of Voronoi polygons $V_{i}$ corresponding to $i^{\text {th }}$ node .

$$
\begin{aligned}
& \text { If } \mathrm{V}_{\mathrm{i}}<<<\mathrm{pi}\left(\mathrm{S}_{\mathrm{R}}\right)^{2} \\
& \mathrm{Di}=\text { " high" } \\
& \text { Else } \\
& \mathrm{Di}=" \text { low" }
\end{aligned}
$$

Step-3:

// Rank of $i^{\text {th }}$ node: Rank $k_{i}=E_{i} / E_{T}$

$E_{i}$ energy left with $i^{\text {th }}$ node

$E_{T}$ Threshold energy specified//

If $\quad$ Rank $_{\mathrm{i}}<1$

Else

//Node $\mathrm{i}$ is in equilibrium (no movement)//

// Vertices of Voronoi polygon $\mathrm{V}_{1}\left(\mathrm{~V}_{11}, \mathrm{~V}_{12}, \mathrm{~V}_{13 \ldots} \ldots\right)$

Distance between node's current position \& the Voronoi polygon is calculated// .

for $(i=1 ; i<N ; i++)$

\{

// node 'i's movement//

$\mathrm{d}_{\mathrm{i}, \mathrm{j}}=\operatorname{sqrt}\left[\left(\mathrm{V}_{\mathrm{xj}}-\mathrm{x}_{0}\right)^{2}+\left(\mathrm{V}_{\mathrm{yj}}-\mathrm{y}_{0}\right)\right]$

$\mathrm{d}_{\mathrm{i}}=\max \left(\mathrm{d}_{\mathrm{i}, \mathrm{j}}\right)$

//move the node towards $V_{i, j}$ /

Research article

(C)Indian Society for Education and Environment (iSee)
\}

Go to step 2;

Results and discussion

Here a square area of $\left(100^{*} 100\right) \mathrm{m}^{2}$ is considered as the area of interest with 20 specified mobile wireless sensor nodes. The initial deployment of nodes in the area of interest is done randomly as shown in Fig. 3(a).

Fig. 3(b) shows more uniformly distributed nodes compared to Fig. 3(a). The difference might be due to the use of rank in the proposed algorithm. As in this scheme the movement of nodes is caused by neighboring nodes and Voronoi vertex, the Voronoi diagram corresponding to initial deployment in Fig. 3(a) is shown in Fig. 4(a) in which the area of the polygons are different from each other; but using our proposed algorithm the area of polygons become almost closer to each other as shown in Fig.4 (b). Additionally, the energy consumption of the whole network with distance is plotted in Fig.5. Compare to random deployment, the energy consumption decreases significantly in Fig. 5(b) over Fig. 5(a), as per our proposed scheme. The energy of individual nodes also decreases noticeably with our proposed scheme compared to the random deployment Fig.6 (a), (b).

\section{Conclusion}

In this work we considered the area of voronoi polygon for initialization step and Rank for the movement step. This Rank based self-deployment scheme of mobile sensors minimizes the energy consumption in a given Area of Interest. The proposed scheme utilizes the repulsive force generated by the virtual potential field due to obstacles and neighboring nodes and the force of attraction generated by the farthest vertex of the voronoi polygon. Energy content is monitored at a certain interval of time to stop the movement of nodes thus one by one all nodes come to an equilibrium. The main advantage of this scheme is that, no node failure occurs while the whole network is in working condition. With our proposed scheme, the energy consumption is much lower with a good coverage area percentage, obtained within a shorter time span compared to other random mobility schemes.

\section{Future scope}

This work can be extended further by making the system adaptive for unexpected obstacles or random occurrences of obstacles in the environment.

\section{Reference}

1. Cardei M and Du DZ (2005) Improving wireless sensor network lifetime through power aware organization. ACM Wireless Networks. 11(3), 333340.

2. Cardei $M$ and $\mathrm{Wu} J$ (2006) Energy-efficient coverage problems in wireless ad hoc sensor networks. Comput. Commun. J. (Elsevier). 29(4), 413-420.

3. Carle J and D Simplot (2004) Energy efficient area monitoring by sensor networks. IEEE Comput. 37(2), 40-46.
"Wireless sensor networks" http://www.indjst.org
Archana Mishra et al. Indian J.Sci.Technol. 
4. Howard A, Mataric MJ and Sukhatme GS (2002) Mobile sensor network deployment using potential fields: A distributed, scalable solution to the area coverage problem. $6^{\text {th }} / \mathrm{nt}$. Conf. Distributed Autonomous Robotic Systems (DSRS02), Fukuoka, Japan. pp: 299-308.

5. Meguerdichian $S$, Koushanfar $F$, Potkonjak $M$ and SrivastavaM (2001) Coverage problems in wireless Ad-hoc sensor networks. IEEE Infocom. pp: 13801387.

6. Nojeong Heo and Pramod K Varshney (2005) Energy-efficient deployment of intelligent mobile sensor networks. IEEE Trans. Sys. Man, \& Cybernetics. Part A: Sys. \& Humans. 35(1), 78-92.

7. Sameera Poduri and Gaurav S Sukhatme (2004) Constrained coverage for mobile sensor Networks. IEEE Int. Conf. Robotics \& Automation. April 26-May 1, 2004, New Orleans, LA, USA. pp: 165-172.

8. Shafaq Chaudhary, Victor Hung and Ratan Guha (2006) Optimal placement of wireless sensor nodes with fault tolerance and minimal energy consumption.2006 IEEE.

9. Wang G, Cao G and La Porta T (2004) Proxy-based sensor deployment for mobile sensor networks. Proc. $1^{\text {st }}$ IEEE Int. Conf. Mobile Ad-hoc \& Sensor Sys. (MASS'04), Fort Lauderdale, Florida, October 2004. 